\title{
25 Research Square \\ Identify Superior Parental Lines for Biparental \\ Crossing via Genomic Prediction: Rice as an \\ Example
}

Ping-Yuan Chung

National Taiwan University

Chen-Tuo Liao ( $\nabla$ ctliao@ntu.edu.tw )

Southern Taiwan University https://orcid.org/0000-0001-9777-3701

\section{Original article}

Keywords: genomic prediction, genomic selection, mixed effects model, rice breeding

Posted Date: August 11th, 2020

DOI: https://doi.org/10.21203/rs.3.rs-53364/v1

License: (c) (1) This work is licensed under a Creative Commons Attribution 4.0 International License.

Read Full License 
7 Background: A set of superior parental lines is the key to high-performing recombinant
Identify Superior Parental Lines for Biparental Crossing via Genomic Prediction: Rice as an Example

Ping-Yuan Chung and Chen-Tuo Liao *
Affiliations: PY Chung and CT Liao, Department of Agronomy, National Taiwan

University, Taipei, Taiwan. *Corresponding author (ctliao@ntu.edu.tw)

\section{ABSTRACT}

inbred lines (RILs) for biparental crossing in a rice breeding program. The number of possible crosses in such a breeding program is often far greater than the number that breeders can handle in the field. A practical parental selection method via genomic prediction (GP) is therefore developed to help breeders identify a set of superior parental lines from a candidate population before field trials.

Results: The parental selection via GP often involves truncation selection, selecting the top fraction of accessions based on their genomic estimated breeding values (GEBVs). However, the truncation selection inevitably causes a loss of genomic diversity in the breeding population. To preserve genomic variation, the selection of closely related accessions should be avoided. We first proposed a new index to quantify the genomic diversity for a set of candidate accessions. Then, we compared the performance of three classes of strategy for the parental selection, including those consider (a) GEBV only, (b) genomic diversity only, and (c) both GEBV and genomic diversity. We analyzed two rice (Oryza sativa L.) genome datasets for the comparison. The results show that the strategies considering both GEBV and genomic diversity have the best or second-best performance for all the traits analyzed in this study.

Conclusion: Combining GP with Monte Carlo simulation can be a useful means of parental selection for rice pre-breeding programs. Different strategies can be implemented to identify a set of superior parental lines from a candidate population. In consequence, the strategies considering both GEBV and genomic diversity that can balance the starting GEBV average with maintenance of genomic diversity should be 
recommended for practical use.

30 Keywords: genomic prediction, genomic selection, mixed effects model, rice breeding.

\section{BACKGROUND}

Biparental crossing is a commonly used scheme in pure-line breeding for self-pollinated crops such as rice, wheat (Triticum aestivum L.), soybean [Glycine max (L.) Merr.] and oat (Avena sativa L.). Plant breeders cross two inbred parental lines to produce $\mathrm{F}_{1}$ population, then a subset of diverse individuals of the $F_{2}$ population is selected to produce potential RILs after several generations of selfing. Obviously, the parental lines play a fundamental role in the line development and significantly affect the performance of the resulting RILs. However, the identification of superior parental lines from germplasm collections for creating genetic variation to maximize selection response in subsequent cycles is still a challenge for plant breeders (Bernardo 2003; Witcombe et al. 2013). Another practical concern is that the number of possible crosses in such a breeding program is often far greater than the number that breeders can handle in the field. Therefore, it should be of great help to breeders if a limited number of superior parents can be identified before the field trial.

Genomic selection based on the statistical method of GP has been used to improve breeding efficiency in dairy cattle (Hayes et al. 2009) and a variety of crops (Massman et al. 2013; Asoro et al. 2011; Heffner et al. 2011; Lorenz et al. 2012; Spindel et al. 2015). The main concept of GP is to capture all the effects of quantitative trait loci (QTLs) by using dense DNA markers over the whole genome, assuming that the DNA markers are in strong linkage disequilibrium with one or more QTLs (Meuwissen et al. 2001). The most commonly used DNA markers are single nucleotide polymorphisms (SNPs). A GP model is first built using the phenotype and genotype data of a training population. Then, GEBVs for the candidate individuals with known genotype data are predicted through the resulting GP model. There are two kinds of mixed linear model methods are widely employed to obtain the GEBVs: (i) best linear unbiased prediction 
the BLUP of (i), the marker effects are treated as random effects and the GEBVs of individuals are calculated by multiplying their marker scores by these BLUP estimates.

60 Ridge regression BLUP (rr-BLUP) method (Meuwissen et al. 2001; Piepho 2009) follows this approach. For the BLUP of (ii), the genotypic values of individuals are treated as random effects and estimated through a genomic relationship matrix. The genomic BLUP (GBLUP) method (Habier et al. 2007; VanRaden 2008) follows this approach. For more details regarding the GP models and the estimation methods used for their model parameters, refer to Xavier et al. (2016).

Gaynor et al. (2017) proposed a two-part strategy for implementing genomic selection for line development, addressing the two components: (i) a product development component, to identify inbred lines either for hybrid parent development or cultivar release; (ii) a population improvement, to increase the frequency of favorable alleles through rapid recurrent genomic selection. Conducting a stochastic simulation, they showed that programs using the two-part strategy generated up to 2.5 times more genetic gain than conventional programs, and up to 1.5 times more genetic gain than the best performing standard genomic selection strategy. Also, Yao et al. (2018) combined GP with Monte Carlo simulation to select superior parents in wheat breeding before the field trial. They used the criterion of usefulness function on a selection index, incorporating yield and two quality traits, to evaluate a cross. Their usefulness function took into account both the mean genetic value and genetic variance of progeny populations. Yao et al. (2018) simulated the required progeny populations using the R/qtl package (Broman et al. 2003), and calculated their usefulness function estimates. It was concluded that the use of the usefulness function for parental selection resulted in higher genetic gain than the use of mid-parent GEBV, implying that the strategy for the parental selection cannot only consider GEBVs of the candidate accessions.

Selecting the parental lines with the highest GEBVs (truncation selection), breeders hope to maximally pass favorable properties of the parental lines on to their progeny populations. However, several favorable QTLs can risk being eliminated from the breeding population using the truncation selection (Vanavermaete et al. 2020). We therefore take both GEBV and genomic diversity into account for identifying superior 
89 GBLUP model to predict the GEBVs for the candidate accessions. Furthermore, we

90 propose a new index to quantify the genomic diversity for a set of candidate accessions 91 according to the GBLUP model. We simulate the genotype data for progeny populations over successive generations derived from a cross between two parental lines. The GEBVs of the progeny populations are then predicted by the trained GBLUP model. We further make generation advancement decisions according to the resulting GEBVs. Finally, we assess a set of parental lines based on their $F_{10}$ RILs which are assumed to be a fixed population. Several selection strategies are evaluated within two rice genome datasets.

\section{MATERIALS AND METHODS}

\section{The Rice Genome Datasets}

101 Dataset I: We first used the rice genome dataset presented in Zhao et al. (2011) to 102 illustrate our proposed procedure. This dataset was originally collected for 103 genome-wide association study (GWAS). The dataset contains 44,100 SNP variants and 10436 traits of 413 O. sativa accessions, and has a strong subpopulation structure 105 containing six different groups. We deleted any SNPs with a missing rate of $>0.05$ and 106 a minor allele frequency of $<0.05$. To reduce redundant collinearity in calculation of the 107 genomic relationship matrix, we only retained about one-third of the SNPs which are 108 evenly distributed over each chromosome. We then imputed a missing SNP marker from 109 its corresponding major homozygous alleles. The final marker matrix consists of 413 110 accessions and 11,047 SNPs. We here analyzed the six traits: brown rice seed width 111 (BRSW), florets per panicle (FPP), flowering time at Arkansas (FTAA), flowering time 112 at Faridpur (FTAF), plant height (PH), and panicle number per plant (PNPP).

113 Dataset II: We further analyzed the rice genome dataset presented in Spindle et al. 114 (2015), which was collected for genomic selection study. The dataset contains 73,147

115 SNP variants and 363 elite breeding lines belonging to indica or indica-admixed group. 116 The phenotype data include the four years (2009-2012), two seasons per year (dry and 
117 wet), of grain yield (YLD), flowering time (FT), and plant height (PH). Note that the

118 PH data in 2009 wet season are not available. The adjusted means for 328 out of the 363

119 individuals and 10,772 out of the 73,147 SNP markers were used for this study. We here

120 chose one marker every $0.1 \mathrm{cM}$ over each chromosome.

121 Monte Carlo Simulation for the Genotype of Progeny Populations

122 To simulate the genotype data for progeny populations, we used Gramene Annotated

123 Nipponbare Sequence (Youens-Clark et al. 2011) to estimate recombination rates

124 between two adjacent SNPs. The Gramene Annotated Nipponbare Sequence database

125 contains both the physical and linkage distances between SNPs, which can be

126 downloaded from http://archive.gramene.org. The genetic positions of the SNPs are

127 estimated via linear interpolation between the two markers flanking each SNP. Once the

128 genetic positions were obtained, the recombination rates between adjacent SNPs were

129 estimated via Haldane's mapping function (Haldane 1919):

$$
r_{A B}=\frac{1}{2}\left(1-e^{-2 X_{A B}}\right),
$$

131 where $r_{A B}$ is the recombination rate and $X_{A B}$ is the linkage distance between SNP markers A and B. Through a series of Bernoulli distributions and the estimated recombination rates, the crossover of each chromosome was simulated to yield the sequence of a gamete, then two gametes were paired to produce the genotype data for the progeny.

\section{GBLUP Model}

137 We considered the following single-trait GBLUP model for GP:

$$
\boldsymbol{y}=\mu \mathbf{1}_{n}+\boldsymbol{g}+\boldsymbol{e},[1]
$$

139 where $\mathbf{y}$ denotes the vector of phenotypic values of a training population with $n$

140 individuals; $\mu$ is a constant term; $\mathbf{1}_{n}$ is the vector of order $n$ with all elements equal to

$141 \quad 1 ; \boldsymbol{g}$ stands for the vector of genotypic values and $\boldsymbol{e}$ is the vector of random errors. It

142 is assumed that $\boldsymbol{g}$ follows a multi-variate normal distribution $\operatorname{MVN}\left(\mathbf{0}, \sigma_{g}^{2} \boldsymbol{K}\right)$, where $\mathbf{0}$ 
143 is a zero vector; $\sigma_{g}^{2}$ is the genetic variance of additive effects and $\boldsymbol{K}$ is a genomic

144 relationship matrix among the individuals. Furthermore, $\boldsymbol{e}$ follows $\operatorname{MVN}\left(\mathbf{0}, \sigma_{e}^{2} \boldsymbol{I}_{n}\right)$,

145 where $\sigma_{e}^{2}$ is the random error variance and $\boldsymbol{I}_{n}$ denotes the identity matrix of order $n$.

146 Here, $\boldsymbol{g}$ and $\boldsymbol{e}$ are assumed to be mutually independent. In this study, we considered

147 the genomic relationship matrix $\boldsymbol{K}=\boldsymbol{M} \boldsymbol{M}^{T} / p$, where $\boldsymbol{M}$ is the marker score matrix

148 and $p$ is the number of SNP markers. The elements of $\boldsymbol{M}$ are coded as $-1,0$, and 1 for

149 the minor homozygous alleles $\left(A_{1} A_{1}\right)$, the heterozygous alleles $\left(A_{1} A_{2}\right)$, and the major

150 homozygous alleles $\left(A_{2} A_{2}\right)$, respectively. The model parameters of the GBLUP model

151 can be estimated through Henderson's equations (Henderson 1984), given by:

$$
\left[\begin{array}{cc}
n & \mathbf{1}_{n}^{T} \\
\mathbf{1}_{n} & \boldsymbol{I}_{n}+\lambda \boldsymbol{K}^{-1}
\end{array}\right]\left[\begin{array}{c}
\hat{\mu} \\
\widehat{\boldsymbol{g}}
\end{array}\right]=\left[\begin{array}{c}
\mathbf{1}_{n}^{T} \boldsymbol{y} \\
\boldsymbol{y}
\end{array}\right],[2]
$$

153 where the regularization parameter $\lambda$ is given by $\lambda=\frac{\sigma_{e}^{2}}{\sigma_{g}^{2}}$. We used the $\mathrm{R}$ function 154 mmer( ) in the R package sommer (Covarrubias-Pazaran 2016) to obtain the restricted 155 maximum likelihood estimates (REMLs) for the two variance components of $\sigma_{g}^{2}$ and $156 \sigma_{e}^{2}$, and then plugged the resulting estimates into Eq. [2] to get $\hat{\mu}$ and $\widehat{\boldsymbol{g}}$.

Let $\widehat{\boldsymbol{g}}_{b p}$ be the vector of estimated genotypic values for a breeding population and $158 \boldsymbol{K}_{b p}$ be the genomic relationship matrix between the breeding population and the 159 training population. In the case, we have:

$$
\widehat{\boldsymbol{g}}_{b p}=\boldsymbol{K}_{b p} \boldsymbol{K}^{-1} \widehat{\boldsymbol{g}} .
$$

161 The GEBV for the breeding population is $\widehat{\boldsymbol{g}}_{b p}$ plus the estimate of the constant term $\hat{\mu}$.

\section{The Index to Quantify Genomic Diversity}

163 Let $\boldsymbol{g}_{0}$ be the vector of genotypic values and $\boldsymbol{K}_{0}$ be the genomic relationship matrix 164 for a particular set of accessions with size $n_{0}$. According to the GBLUP model of Eq. 165 [1], the covariance matrix for $\boldsymbol{g}_{0}$ is given by:

$$
\operatorname{Var}\left(\boldsymbol{g}_{0}\right)=\sigma_{g}^{2} \boldsymbol{K}_{0}
$$

167 The determinant of the covariance matrix represents the overall variability for the 
genotypic values, which is calculated as:

$$
\left|\operatorname{Var}\left(\boldsymbol{g}_{0}\right)\right|=\left(\sigma_{g}^{2}\right)^{n_{0}}\left|\boldsymbol{K}_{0}\right| .[3]
$$

170 Clearly, the determinant of Eq. [3] is proportional to the $D$-score defined below:

$$
D \text {-score }=\left|\boldsymbol{K}_{0}\right| .[4]
$$

172 The $D$-score of Eq. [4] ranges from 0 to 1 . For a fixed number of $n_{0}$, a subset of 173 accessions chosen from a breeding population that achieves the maximal D-score will 174 have greater genomic diversity than the competing choices with size $n_{0}$. The concept of 175 the $D$-score is adopted from optimum experimental designs (Atkinson and Donev 1992). 176 A simple example is given to illustrate the $D$-score. Suppose that there are $n=3$ accessions in the candidate set with the genomic relationship matrix:

$$
\boldsymbol{K}=\left[\begin{array}{ccc}
1 & 0.7 & 0.5 \\
0.7 & 1 & 0.3 \\
0.5 & 0.3 & 1
\end{array}\right]
$$

179 For $n_{0}=2$, the $D$-score for $g_{1}$ and $g_{2}$ is calculated as $\left|\boldsymbol{K}_{0}\right|=\left|\begin{array}{cc}1 & 0.7 \\ 0.7 & 1\end{array}\right|=0.51$.

180 Similarly, the $D$-scores for $g_{1}$ and $g_{3}$, and for $g_{2}$ and $g_{3}$ are given by 0.75 and 0.91 , 181 respectively. Clearly, the two accessions with $g_{2}$ and $g_{3}$ have greater genomic 182 variation (smaller genomic correlation) than the other competing choices. A set of 183 accessions with the maximal D-score can avoid the selection of closely related 184 individuals.

\section{An Algorithm to Search for Accessions with the Maximal $D$-Score}

186 We required a highly efficient algorithm to search for a subset of accessions within a candidate population so that it can achieve the maximal $D$-score. We used a genetic algorithm to complete this task, which is an exchange algorithm with the three different operators: roulette wheel selection, crossover, and mutation (Whitley 1994). For a given

190 candidate set $S_{c}$ with $n_{c}$ accessions, we searched for an optimal subset $S_{0}$ with $n_{0}$

191 individuals from $S_{c}$. Our algorithm began with a set of $m$ random solutions, each of 192 which is a vector of 0 or 1 with a length equal to $n_{c}$. The number of values with a score 
193 of 1 in the vector is equal to $n_{0}$, corresponding to the chosen accessions at the current 194 stage. Here, we fixed $m=n_{0}$. We then obtained the elite solutions from the initial $m$ 195 random solutions after a large number of iterations, where each iteration repeated all the 196 three operators. We stopped the algorithm when the maximal $D$-score among the current 197 elite solutions converged.

\section{The Procedure for Selecting Parental Lines}

199 To evaluate a variety of strategies in determining parental lines, we carried out the 200 following steps.

201 Step 1: For a specific target trait, we used all of the phenotypic values available from 202 the rice genome dataset to build the corresponding GBLUP model of Eq. [1].

203 Step 2: We predicted the GEBVs for all of the accessions in the dataset through the 204 trained GBLUP model developed in Step 1. Seven strategies were used to select a subset of 10 parental lines according to their GEBVs: (i) the GEBV only (GEBV-O) approach, which chose the top 10 accessions (either maximal or minimal); the genomic diversity only (GD-O) approaches: (ii) GD-O-30, (iii) GD-O-50, and (iv) GD-O-100, which applied the genetic exchange algorithm to search for an optimal subset of 10 accessions from each of the three candidate sets composed of the top 30, 50, and 100 accessions,

210 respectively, such that the chosen subset had the maximal $D$-score; and the approaches

211 (GEBV-GD) considering both GEBV and genomic diversity: (v) GEBV-GD-30, (vi) 212 GEBV-GD-50, and (vii) GEBV-GD-100, which retained the top two accessions, then

213 applied the genetic exchange algorithm to search for another eight accessions from the 214 remainder of each candidate set for GD-O-30, GD-O-50, and GD-O-100, respectively, 215 so that the resulting 10 accessions had the maximal $D$-score.

216 Step 3: For each subset of 10 accessions determined by the seven strategies, we crossed

217 any two parental lines to produce $45 \mathrm{~F}_{1}$ hybrids. Here, we started to simulate the 218 genotype data for successive generations of progeny populations through the Monte 219 Carlo simulation. Each of the $45 \mathrm{~F}_{1}$ hybrids produced 60 individuals of the $\mathrm{F}_{2}$ 220 population by self-pollination, resulting in $2700 \mathrm{~F}_{2}$ individuals. After obtaining the 221 GEBVs for the $2700 \mathrm{~F}_{2}$ individuals via the trained GBLUP model of Step 1, we then 
222 retained the top $45 \mathrm{~F}_{2}$ individuals. Again, we used these $45 \mathrm{~F}_{2}$ individuals to produce

$2232700 \mathrm{~F}_{3}$ individuals (each $\mathrm{F}_{2}$ individual produced $60 \mathrm{~F}_{3}$ individuals) and retained the top

$22445 \mathrm{~F}_{3}$ individuals. We then repeated the same procedure to produce $2700 \mathrm{~F}_{10}$ individuals 225 which are assumed to be a fixed population.

226 Step 4: For the resulting $2700 \mathrm{~F}_{10}$ individuals generated according to each strategy, we 227 found the best $\mathrm{F}_{10}$ RIL with the top GEBV.

228 A flowchart of the procedure is displayed in Figure 1. We repeated this analysis 229 procedure 30 times to obtain the best $F_{10}$ RILs from each repetition for each strategy.

230 The average of the GEBVs for the best $F_{10}$ RILs was then calculated and used as the 231 measure of efficiency for the strategy. Note that for the traits of BRSW, FPP, and PNPP 232 in Dataset I; and YLD in Dataset II, larger GEBVs are preferable (i.e., these traits 233 follow the rule that the larger, the better). The remaining five traits of FTAA, FTAF, and 234 PH in Dataset I; and FT, and PH in Dataset II are those for which the rule is "the smaller, 235 the better".

\section{Calculation of Genetic Gain}

To gain an understanding of the genetic improvement on a target trait using different strategies, we estimated genetic gain as

$$
\text { genetic gain }=\overline{G E B V}_{F_{10}}-\overline{G E B V}_{P},[5]
$$

240 where $\overline{G E B V}_{F_{10}}$ denotes the GEBV average among the resulting $2700 \mathrm{~F}_{10}$ RILs and

$241 \overline{G E B V}_{P}$ denotes the GEBV average among the 10 selected parental lines for each 242 strategy (Rutkoski 2019). The larger absolute value of the genetic gain indicates the 243 more improvement on the target trait. 


\section{Strategies Comparison Based on the best F $_{10}$ RILs}

The GEBV averages of the best $F_{10}$ RILs from the 30 repetitions using each of the seven strategies are displayed in Tables 1 and 2 for the two datasets. The results in the tables show that the strategies considering both GEBV and genomic diversity

250 (GEBV-GD-30, -50, -100) generally have satisfactory efficiency, because they achieve 251 the best or second-best performance for all the traits. Therefore, this kind of strategies could be a reliable means of determining the parental lines. On the other hand, the strategies accounting for genomic diversity only (GD-O-30, -50, -100) don't have satisfactory efficiency for all the traits, with the exception of GD-O-100 for YLD in Dataset II. For the strategy based on GEBV only, the GEBV-O has the best or second-best performance for FPP, and PH in Dataset I; and PH, and FT in Dataset II, but also has the worst or second-worst performance for the remaining four traits in Dataset I and YLD in Dataset II. Thus, the GEBV-O could be a high-risk strategy.

We also displayed the GEBV averages with the plus and minus one unit of their corresponding standard deviations for the best individuals from the 30 repetitions over consecutive generations in Figures 2 and 3. From the figures, the four strategies of GEBV-O, GEBV-GD-30, -50, -100 selected the same best individual from the 30 repetitions at parental generation, and also at $\mathrm{F}_{1}$ generation, so there is no standard deviation shown with the corresponding GEBV averages. The GEBV averages of the best selected parental lines by the strategies can be ranked as GEBV-O = GEBV-GD-30 $=$ GEBV-GD-50 = GEBV-GD-100 > GD-O-30 > GD-O-50 > GD-O-100 in decreasing desirability. The desirability at parental generation decreases as the degree of diversity increases for the three strategies considering the genomic diversity only. Also, the desirability declines from parental generation to $F_{1}$ generation for every strategy, due to the heterogenous alleles in $F_{1}$ hybrids.

To explore the extent to which the top two accessions contribute to the subset of ten parental lines determined by the four strategies of GEBV-O, GEBV-GD-30, -50, -100 , we compared each subset with a reduced group consisting of $F_{1}$ hybrids whose parental lines contain at least one of the top two accessions for each subset. Every reduced group consists of $17 \mathrm{~F}_{1}$ hybrids. Similarly, we followed the analysis procedure 
276 to obtain the GEBV averages for the best $F_{10}$ RILs from 30 repetitions based on the

277 reduced group. The results are displayed in Table 3 with the corresponding GEBV 278 averages based on the group of the original $45 \mathrm{~F}_{1}$ hybrids. From the table, there is no 279 practical significant difference between these two groups for all the traits using the four 280 strategies.

281 Genetic Gains for the Strategies

282 The average among the genetic gains on a target trait for each strategy calculated 283 by Eq. [5] from the 30 repetitions is displayed in Tables 4 and 5 for Datasets I and II, 284 respectively. It is reasonable to compare the performance of the strategies according to 285 the endpoint of $\overline{G E B V}_{F_{10}}$. From the tables, we found that the comparison results based 286 on $\overline{G E B V}_{F_{10}}$ are consistent with the above results based on the best $\mathrm{F}_{10}$ RILs. Also, the 287 strategies considering genomic diversity (GD-O-30, -50, -100; GEBV-GD-30, -50, -100) 288 have greater genetic gain than the GEBV-O for all the traits except PH in Dataset I 289 (Table 4). As expected, the genetic gain usually increases with the increase of the 290 genomic diversity (GD-O-100 outperforms both GD-O-50 and GD-O-30 for all the 291 traits except BRSW, and FTAF in Dataset I; GEBV-GD-100 outperforms both 292 GEBV-GD-50 and GEBV-GD-30 for all the traits). In addition, GEBV-O has the best $293 \overline{G E B V}_{P}$; GEBV-GD-30 has better $\overline{G E B V}_{P}$ than GD-O-30; GEBV-GD-50 has better $294 \overline{G E B V}_{P}$ than GD-O-50 and GEBV-GD-100 has better $\overline{G E B V}_{P}$ than GD-O-100 for all 295 the traits. Namely, a strategy has a relatively good starting point as it considers more 296 degree of GEBV.

\section{DISCUSSION}

299 From the results for comparing the proposed strategies, those considering both GEBV 300 and genomic diversity or considering GEBV only can be recommended for practical use. 301 Furthermore, from the results for exploring the extent to which the top two accessions 302 contribute to the parental lines determined by the four strategies of GEBV-O, 303 GEBV-GD-30, -50, -100, we have the conclusion: the economical strategies with $17 \mathrm{~F}_{1}$ 
304 hybrids whose parental lines contain at least one of the top two accessions for each 305 selected subset can be a practical alternative to those with $45 \mathrm{~F}_{1}$ hybrids composed of all 306 of the possible crosses.

From Tables 4 and 5, the strategies considering genomic diversity only (GD-O-30, $-50,-100)$ generally have greater genetic gain, mainly due to their more genomic variation but less favorable $\overline{G E B V}_{P}$, so they have more room to improve. Also, the GEBV-O has the best starting $\overline{G E B V}_{P}$ but the least genomic diversity in the base population, so it has less potential to improve. The strategies considering both GEBV and genomic diversity (GEBV-GD-30, -50, -100) could balance the tradeoff between starting $\overline{G E B V}_{P}$ and genomic variation of the base population.

Dataset II was specifically collected for genomic selection. All of the available accessions in the dataset belong to indica or indica-admixed group. From the results of the performance based on the best $\mathrm{F}_{10}$ RILs in Table 2, all the seven strategies seem to have close performance for the three target traits. The resulting GEBV averages of the best $F_{10}$ RILs range from 6472 to $6546 \mathrm{~kg} / \mathrm{ha}$ for YLD, from 85.889 to $91.852 \mathrm{~cm}$ for $\mathrm{PH}$, and from 77.725 to 78.410 days for FT. This could be due to the fact that the candidate accessions in Dataset II are elite breeding lines which have limited genomic diversity and similar phenotypic values for the target traits. However, the two strategies with greater genomic diversity, GD-O-100 and GEBV-GD-100 for YLD (their corresponding GEBV averages are 6546 and $6539 \mathrm{~kg} / \mathrm{ha}$ ), led to larger YLD than the other five strategies (their corresponding GEBV averages range from 6472 to 6506 $\mathrm{kg} / \mathrm{ha}$ ). The four strategies of GEBV-O, GEBV-GD-30, -50, -100 performed equally well for PH (their corresponding GEBV averages range between 85.817 and $86.062 \mathrm{~cm}$ ), but slightly better than GD-O-30, -50, -100 (their corresponding GEBV averages are $87.517,89.920$, and $91.799 \mathrm{~cm})$. The consistent results based on the $\overline{G E B V}_{F_{10}}$ can be found in Table 5.

It is known that Dataset I contains more genomic diversity than Dataset II, since it consists of five subpopulations and one admixed group. The more genomic diversity of

332 Dataset I could lead to a bigger difference between the strategies considering both 333 GEBV and genomic diversity, and the strategy considering GEBV only for some traits. 
334 For example, the difference of the GEBV averages among the best $F_{10}$ RILs between

335 GEBV-GD-50 and GEBV-O is about -9.06 days for FTAA, and -2.55 days for FTAF in

336 Dataset I (Table 1), but the corresponding difference is just -0.09 days for FT in Dataset

337 II (Table 2). However, the flowering time is very sensitive to environments, so the

338 genomic diversity cannot solely amount to the different results between these two

339 datasets. More interestingly, the more genomic diversity of Dataset I could lead to a

340 larger genetic gain for a specific trait. From Table 4, the mean of the genetic gains using

341 the seven strategies for PH in Dataset I is given by $-42.15 \mathrm{~cm}$. But, from Table 5, the

342 corresponding mean in Dataset II is just $-13.79 \mathrm{~cm}$.

Daetwyler et al. (2015) and Goiffon et al. (2017) highlighted that an increase in rare favorable alleles in a population can help improve selection responses. Selecting only parental lines with the highest GEBVs can result in a loss of rare favorable alleles for some target traits, thus missing potential RILs over future generations. From the results of BRSW, FTAA, FTAF, and PNPP in Figure 2; and YLD in Figure 3, the performance of GEBV-O appears to be inferior to GEBV-GD-30, -50, -100. This indicates that an increase in genomic diversity in parental lines could compensate for this possible deficiency, and then improve the long-term response to the target traits. The greater genomic diversity could increase the possibility of containing favorable alleles in parental lines, and we therefore expect that the chance of harboring the favorable alleles would increase in RIL populations.

Apparently, the numbers of accessions fixed in the proposed strategies seem to be a little arbitrary, such as those of selecting 10 parental lines, retaining the top 2 accessions, and searching 10 or another 8 accessions from the three candidate sets composed of the top 30, 50 and 100 accessions, respectively. A user certainly can adjust these numbers in the strategies for her/his own study. Also, it was required to have historical phenotypic data used to build the GP model. If the historical phenotypic data are not available, then a pilot experiment is needed to phenotype a set of accessions, which can be determined using an optimization algorithm (Ou and Liao 2019). An R function for performing the proposed procedure of selecting parental lines is available from the authors upon request. 
As mentioned earlier, Yao et al. (2018) evaluated genetic gain using the usefulness function in parental selection, and showed that their selection strategy outperformed the strategy using the mid-parent GEBV. In this study, we emphasized both GEBV and genomic diversity in parental selection, and we made generation advancement decisions for each selection strategy according to the GEBVs of the top individuals. Finally, we compared different strategies based on the performance of the best $F_{10}$ RILs, and discussed genetic gain for target traits using the strategies. Moreover, Yao et al. (2018) showed that applying a selection index incorporating multiple traits can simultaneously improve both yield and quality in wheat than the individual trait selection. Also, Jia and Jannink (2012), Hayashi and Iwata (2013), and Guo et al. (2014) highlighted that multiple-trait GP models can provide better prediction accuracy than single-trait GP models for those traits with low heritability. We will consider the selection index and the multiple-trait GP models into the framework of the current study, so as to investigate the multiple-trait situations in a future study.

\section{CONCLUSIONS}

380 Combining GP with Monte Carlo simulation can be a useful means of detecting superior

381 parents for rice pre-breeding programs. Different strategies can be implemented to 382 identify a set of superior parental lines from a candidate population. The strategy 383 considering GEBV only can have a better starting GEBV average but less genomic 384 diversity in the base population. On the other hand, the strategies considering genomic 385 diversity only can have greater genomic diversity but a less favorable starting GEBV 386 average in the base population. The strategies considering both GEBV and genomic 387 diversity that can balance the starting GEBV average with maintenance of genomic 388 diversity should be recommended for practical use.

390 Abbreviations: BLUP, best linear unbiased predictor; BRSW, brown rice seed width; 391 GBLUP, genomic best linear unbiased predictor; GEBV, genomic estimated breeding 
392 value; GEBV-GD, algorithms considering both GEBV and genomic diversity; GEBV-O, 393 algorithms considering GEBV only; FPP, florets per panicle; FT, flowering time; FTAA, 394 flowering time at Arkansas; FTAF, flowering time at Faridpur; GD-O, algorithms 395 considering genomic diversity only; PH, plant height; PNPP, panicle number per plant; 396 RIL, recombinant inbred line; SNP, single nucleotide polymorphism; YLD, grain yield.

\section{DECLARATIONS}

401 Ethical Approval and Consent to participate: Not applicable.

402 Consent for publication: Not applicable.

403 Availability of supporting data: Not applicable.

404 Competing interests: The authors declare that there is no conflict of interest.

405 Funding: Not applicable.

406 Authors' contributions: PY analyzed the datasets, wrote the R functions, prepared the 407 tables and figures, and drafted the manuscript. CT supervised the research, derived the 408 analysis approach, and drafted the manuscript.

409 Acknowledgements: This research was supported by the Ministry of Science and 410 Technology, Taiwan (grant number: MOST 108-2118-M-002-002-MY2).

\section{REFERENCES}

414 Asoro, F.G., M.A. Newell, W.D. Beavis, M.P. Scott, and J.L. Jannink. 2011. Accuracy and training population design for genomic selection on quantitative traits in elite north American oats. Plant Genome 4: 132-144.

417 Atkison, A.C., and A.N. Donev. 1992. Optimum experimental designs. New York: 
Bernardo, R. 2003. Parental selection, number of breeding populations, and size of each population in inbred development. Theor. Appl. Genet. 107: 1252-1256.

Broman, K.W., H. Wu, S. Sen, and G.A. Churchill. 2003. R/qtl: QTL mapping in experimental crosses. Bioinformatics 19: 889-890.

Covarrubias-Pazaran, G. 2016. Genome-assisted prediction of quantitative traits using the R package sommer. PLOS One 11: e0156744.

Daetwyler, H.D., M.J. Hayden, G.C. Spangenberg, and B.J. Hayes. 2015. Selection on optimal haploid value increases genetic gain and preserves more genetic diversity relative to genomic selection. Genetics 200: 1341-1348.

Gaynor, R.C., G. Gorjanc, A.R. Bentley, E.S. Ober, P. Howell, R. Jackson, et al. 2017. A two-part strategy for using genomic selection to develop inbred lines. Crop Sci. 57: 2372-2386.

Goiffon, M., A. Kusmec, L. Wang, G. Hu, and P.S. Schnable. 2017. Improving response in genomic selection with a population-based selection strategy: Optimal population value selection. Genetics 206: 1675-1682.

Guo, G., F. Zhao, Y. Wang, Y. Zhang, L. Du, and G. Su. 2014. Comparison of single-trait and multiple-trait genomic prediction models. BMC Genetics 15: 30.

Habier, D., R.L. Fernando, and J.C.M. Dekkers. 2007. The impact of genetic relationship information on genome-assisted breeding values. Genetics 177: 2389-2397.

Haldane, J.B.S. 1919. The combination of linkage values and the calculation of distance between the loci for linked factors. Genetics 8: 299-309.

Hayashi, T., and H. Iwata. 2013. A Bayesian method and its variational approximation 
for prediction of genomic breeding values in multiple traits. BMC bioinformatics 14: 1-14.

Hayes, B.J., P.J. Bowman, A.J. Chamberlain, and M.E. Goddard. 2009. Genomic selection in dairy cattle: Progress and challenges. J. Dairy Sci. 92: 433-443.

Heffner, E.L., J.L. Jannink, and M.E. Sorrells. 2011. Genomic selection accuracy using multifamily prediction models in wheat breeding program. Plant Genome 4: 6575.

Henderson, C.R. 1984. Applications of linear models in animal breeding. Univ. of Guelph, Guelph, Ontario.

Jia, Y., and J.L. Jannink. 2012. Multiple-trait genomic selection methods increase genetic value prediction accuracy. Genetics 192: 1513-1522.

Lorenz, A.J., K.P. Smith, and J.L. Jannink. 2012. Potential and optimization of genomic selection for Fusarium head blight resistance in six-row barley. Crop Sci. 52: $1609-1621$.

Massman, J.M., H.J.G. Jung, and R. Bernardo. 2013. Genomewide selection versus marker-assisted recurrent selection to improve grain yield and stover-quality traits for cellulosic ethanol in maize. Crop Sci. 53: 58-66.

Meuwissen, T.H.E., B.J. Hayes, and M.E. Goddard. 2001. Prediction of total genetic value using genome-wide dense marker maps. Genetics 157: 1819-1829.

Ou, J.H., and C.T. Liao. 2019. Training set determination for genomic selection. Theor.

Piepho, H.P. 2009. Ridge regression and extensions for genome-wide selection in maize. Crop Sci. 49: 1165-1176.

Rutkoski, J.E. 2019. A practical guide to genetic gain. Adv. Agron. 157: 217-249. 
Spindel, J., H. Begum, D. Akdemir, P. Virk, B. Collard, E. Redona, et al. 2015. Genomic selection and association mapping in rice (Oryza sativa): Effect of trait genetic architecture, training population composition, marker number and statistical model on accuracy of rice genomic selection in elite, tropical rice breeding lines. PLOS Genetics 11: e1005350.

VanRaden, P.M. 2008. Efficient methods to compute genomic predictions. J. Dairy Sci. 91: 4414-4423.

Vanavermaete, D., J. Fostier, S. Maenhout, and B. De Baets. 2020. Preservation of genetic variation in a breeding population for long-term genetic gain. G3: Genes|Genomes|Genetics doi:10.1534/g3.120.401354.

Whitley, D. 1994. A genetic algorithm tutorial. Stat. Comput. 4: 65-85.

Witcombe, J.R., S. Gyawali, M. Subedi, D.S. Virk, and K.D. Joshi. 2013. Plant breeding can be made more efficient by having fewer, better crosses. BMC Plant Biol. 13: 22.

Xavier, A., W.M. Muir, B. Craig, and K.M. Rainey. 2016. Walking through the statistical black boxes of plant breeding. Theor. Appl. Genet. 129: 1933-1949.

Yao, J., D. Zhao, X. Chen, Y. Zhang, and J. Wang. 2018. Use of genomic selection and breeding simulation in cross prediction for improvement of yield and quality in wheat (Triticum aestivum L.). Crop J. 6: 353-365.

Youens-Clark, K., E. Buckler, T. Casstevens, C. Chen, G. DeClerck, P. Derwent, et al. 2011. Gramene database in 2010: updates and extensions. Nucleic Acids Res. 39: D1085-D1094.

Zhao, K., C.W. Tung, G.C. Eizenga, M.H. Wright, M.L. Ali, A.H. Price, et al. 2011. Genome-wide association mapping reveals a rich genetic architecture of complex traits in Oryza sativa. Nature Communications 2: 467. 
Table 1: The ranking and the GEBV average (in parentheses) for the best $F_{10}$ RILs from 497 the 30 repetitions using the seven proposed strategies in Dataset I.

498

\begin{tabular}{lcccccc}
\hline & BRSW & FPP & FTAA & FTAF & PH & PNPP \\
\hline GEBV-O & $\underline{6(3.418)}$ & $\mathbf{2 ( 5 . 9 6 1 )}$ & $\underline{6(56.521)}$ & $\underline{6(61.856)}$ & $\mathbf{1 ( 4 2 . 1 8 5 )}$ & $\underline{6(4.125)}$ \\
GD-O-30 & $\underline{7(3.408)}$ & $5(5.951)$ & $3(51.564)$ & $3(59.353)$ & $5(49.337)$ & $3(4.188)$ \\
GD-O-50 & $3(3.576)$ & $\underline{6(5.916)}$ & $5(53.348)$ & $5(60.126)$ & $\underline{6(49.801)}$ & $5(4.138)$ \\
GD-O-100 & $4(3.496)$ & $\underline{7(5.882)}$ & $\underline{7(56.835)}$ & $\underline{7(61.967)}$ & $\underline{7(51.788)}$ & $\underline{7(4.086)}$ \\
GEBV-GD-30 & $5(3.419)$ & $3(5.954)$ & $\mathbf{1 ( 4 7 . 1 3 6 )}$ & $\mathbf{1 ( 5 9 . 2 1 6 )}$ & $\mathbf{2 ( 4 2 . 6 9 9 )}$ & $\mathbf{1 ( 4 . 2 2 5 )}$ \\
GEBV-GD-50 & $\mathbf{1 ( 3 . 6 5 6 )}$ & $\mathbf{1 ( 5 . 9 6 4 )}$ & $\mathbf{2 ( 4 7 . 4 5 7 )}$ & $\mathbf{2 ( 5 9 . 3 0 4 )}$ & $3(43.232)$ & $\mathbf{2 ( 4 . 2 1 4 )}$ \\
GEBV-GD-100 & $\mathbf{2 ( 3 . 6 3 4 )}$ & $4(5.953)$ & $4(51.382)$ & $4(59.634)$ & $4(43.498)$ & $4(4.171)$
\end{tabular}

499

500 (i) The best and second-best strategies are indicated in bold text, and the worst and 501 second-worst strategies are indicated by underlining.

502 (ii) GEBV-O: the subset of the top 10 accessions with the minimal or maximal GEBVs;

503 GD-O-30, -50, -100: the subsets of 10 accessions with the maximal D-scores chosen 504 from the candidate sets composed of the top 30, 50, and 100 accessions, respectively; 505 GEBV-GD-30, -50, -100: the subsets of the top 2 accessions plus 8 accessions chosen 506 from the remainder of the candidate sets composed of the top 30, 50, and 100 accessions, 507 respectively, which have the maximal D-scores. 
508 (iii) BRSW: brown rice seed width; FPP: florets per panicle; FTAA: flowering time at 509 Arkansas; FTAF: flowering time at Faridpur; PH: plant height; PNPP: panicle number 510 per plant.

516 Table 2: The ranking and the GEBV average (in parentheses) for the best $\mathrm{F}_{10}$ RILs from 517 the 30 repetitions using the seven proposed strategies in Dataset II.

518

519

\begin{tabular}{llcc}
\hline & YLD & PH & FT \\
\hline GEBV-O & $\underline{7(6472)}$ & $\mathbf{1 ~ ( 8 5 . 8 1 7 )}$ & $\mathbf{2 ( 7 7 . 8 1 8 )}$ \\
GD-O-30 & $4(6491)$ & $5(87.517)$ & $\underline{7(78.410)}$ \\
GD-O-50 & $5(6489)$ & $\underline{6(89.920)}$ & $5(78.164)$ \\
GD-O-100 & $\mathbf{1 ( 6 5 4 6 )}$ & $\underline{7(91.799)}$ & $\underline{6(78.359)}$ \\
GEBV-GD-30 & $3(6506)$ & $\mathbf{2 ( 8 5 . 9 7 6 )}$ & $4(77.883)$ \\
GEBV-GD-50 & $\underline{6(6485)}$ & $3(85.917)$ & $\mathbf{1 ( 7 7 . 7 2 5 )}$ \\
GEBV-GD-100 & $\mathbf{2 ( 6 5 3 9 )}$ & $4(86.062)$ & $3(77.873)$ \\
\hline
\end{tabular}

520 (i) The best and second-best strategies are indicated in bold text, and the worst and

521 second-worst strategies are indicated by underlining.

522 (ii) GEBV-O: the subset of the top 10 accessions with the minimal or maximal GEBVs;

523 GD-O-30, -50, -100: the subsets of 10 accessions with the maximal D-scores chosen

524 from the candidate sets composed of the top 30, 50, and 100 accessions, respectively;

525 GEBV-GD-30, -50,-100: the subsets of the top 2 accessions plus 8 accessions chosen

526 from the remainder of the candidate sets composed of the top 30, 50, and 100 accessions, 
527

528

529

530

531

532

533

534

535

536

537

respectively, which have the maximal D-scores.

(iii) YLD: yield; PH: plant height; FT: flowering time.

(1)

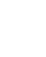

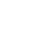

Table 3: The GEBV averages for the best $F_{10}$ RILs from the 30 repetitions based on the group of the original $45 F_{1}$ hybrids and the reduced group of $17 F_{1}$ hybrids using the four strategies of GEBV-O, GEBV-GD-30, GEBV-GD-50, and GEBV-GD-100.

\begin{tabular}{ccccccccc}
\hline & \multicolumn{2}{c}{ GEBV-O } & \multicolumn{2}{c}{ GEBV-GD-30 } & \multicolumn{2}{c}{ GEBV-GD-50 } & \multicolumn{2}{c}{ GEBV-GD-100 } \\
\hline Dataset I & $45 \mathrm{~F}_{1}$ & $17 \mathrm{~F}_{1}$ & $45 \mathrm{~F}_{1}$ & $17 \mathrm{~F}_{1}$ & $45 \mathrm{~F}_{1}$ & $17 \mathrm{~F}_{1}$ & $45 \mathrm{~F}_{1}$ & $17 \mathrm{~F}_{1}$ \\
\hline BRSW & 3.418 & 3.423 & 3.419 & 3.418 & 3.656 & 3.652 & 3.634 & 3.650 \\
FPP & 5.961 & 5.965 & 5.954 & 5.957 & 5.964 & 5.958 & 5.953 & 5.943 \\
FTAA & 56.521 & 57.513 & 47.136 & 46.961 & 47.457 & 47.421 & 51.382 & 51.734 \\
FTAF & 61.856 & 61.850 & 59.216 & 59.123 & 59.304 & 59.232 & 59.634 & 59.713 \\
PH & 42.185 & 43.409 & 42.699 & 43.271 & 43.232 & 43.791 & 43.498 & 43.854 \\
PNPP & 4.125 & 4.129 & 4.225 & 4.226 & 4.214 & 4.204 & 4.171 & 4.161 \\
\hline Dataset II & $45 \mathrm{~F}_{1}$ & $17 \mathrm{~F}_{1}$ & $45 \mathrm{~F}_{1}$ & $17 \mathrm{~F}_{1}$ & $45 \mathrm{~F}_{1}$ & $17 \mathrm{~F}_{1}$ & $45 \mathrm{~F}_{1}$ & $17 \mathrm{~F}_{1}$ \\
\hline YLD & 6472 & 6476 & 6506 & 6499 & 6485 & 6484 & 6539 & 6534 \\
PH & 85.817 & 85.991 & 85.976 & 85.844 & 85.917 & 86.092 & 86.062 & 86.060 \\
FT & 78.818 & 77.834 & 77.883 & 77.750 & 77.725 & 77.778 & 77.873 & 77.690 \\
\hline
\end{tabular}

538

539 (i) GEBV-O: the subset of the top 10 accessions with the minimal or maximal GEBVs;

540 GEBV-GD-30, -50,-100: the subsets of the top 2 accessions plus 8 accessions chosen

541 from the remainder of the candidate sets composed of the top 30, 50, and 100 accessions,

542 respectively, which have the maximal D-scores. 
543 (ii) BRSW: brown rice seed width; FPP: florets per panicle; FTAA: flowering time at

544 Arkansas; FTAF: flowering time at Faridpur; PH: plant height; PNPP: panicle number 545 per plant.

546 (iii) YLD: yield; PH: plant height; FT: flowering time.

547

548

549

550 Table 4: The average of genetic gains from the 30 repetitions for Dataset I.

551

\begin{tabular}{|c|c|c|c|c|c|c|}
\hline & \multicolumn{3}{|c|}{ BRSW } & \multicolumn{3}{|c|}{ FPP } \\
\hline & $\overline{\mathrm{GEBV}}_{\mathrm{P}}$ & $\overline{\mathrm{GEBV}}_{\mathrm{F}_{10}}$ & $\begin{array}{r}\text { genetic } \\
\text { gain }\end{array}$ & $\overline{\mathrm{GEBV}}_{\mathrm{P}}$ & $\overline{\mathrm{GEBV}}_{\mathrm{F}_{10}}$ & $\begin{array}{l}\text { genetic } \\
\text { gain }\end{array}$ \\
\hline GEBV-O & 3.17 & 3.42 & 0.25 & 5.51 & 5.96 & 0.45 \\
\hline GD-O-30 & 3.10 & 3.41 & 0.31 & 5.48 & 5.95 & 0.47 \\
\hline GD-O-50 & 3.00 & 3.57 & 0.57 & 5.41 & 5.91 & 0.50 \\
\hline GD-O-100 & 2.94 & 3.49 & 0.55 & 5.31 & 5.88 & 0.57 \\
\hline GEBV-GD-30 & 3.12 & 3.42 & 0.30 & 5.48 & 5.95 & 0.47 \\
\hline GEBV-GD-50 & 3.04 & 3.65 & 0.61 & 5.43 & 5.96 & 0.53 \\
\hline \multirow[t]{3}{*}{ GEBV-GD-100 } & 3.00 & 3.63 & 0.63 & 5.34 & 5.95 & 0.61 \\
\hline & \multicolumn{3}{|c|}{ FTAA } & \multicolumn{3}{|c|}{ FTAF } \\
\hline & $\overline{\mathrm{GEBV}}_{\mathrm{P}}$ & $\overline{\mathrm{GEBV}}_{\mathrm{F}_{10}}$ & $\begin{array}{r}\text { genetic } \\
\text { gain } \\
\end{array}$ & $\overline{\mathrm{GEBV}}_{\mathrm{P}}$ & $\overline{\mathrm{GEBV}}_{\mathrm{F}_{10}}$ & $\begin{array}{l}\text { genetic } \\
\text { gain }\end{array}$ \\
\hline GEBV-O & 64.30 & 56.57 & -7.73 & 63.45 & 61.87 & -1.58 \\
\hline GD-O-30 & 72.25 & 49.26 & -22.99 & 64.93 & 59.40 & -5.53 \\
\hline GD-O-50 & 75.41 & 53.54 & -21.87 & 65.82 & 60.16 & -5.66 \\
\hline GD-O-100 & 80.01 & 57.00 & -23.01 & 67.34 & 62.01 & -5.33 \\
\hline GEBV-GD-30 & 71.09 & 47.31 & -23.78 & 64.68 & 59.25 & -5.43 \\
\hline GEBV-GD-50 & 72.86 & 47.64 & -25.22 & 65.40 & 59.35 & -6.05 \\
\hline \multirow[t]{3}{*}{ GEBV-GD-100 } & 77.16 & 51.53 & -25.63 & 66.46 & 59.68 & -6.78 \\
\hline & \multicolumn{3}{|c|}{$\mathrm{PH}$} & \multicolumn{3}{|c|}{ PNPP } \\
\hline & $\overline{\mathrm{GEBV}}_{\mathrm{P}}$ & $\overline{\mathrm{GEBV}}_{\mathrm{F}_{10}}$ & $\begin{array}{r}\text { genetic } \\
\text { gain }\end{array}$ & $\overline{\mathrm{GEBV}}_{\mathrm{P}}$ & $\overline{\mathrm{GEBV}}_{\mathrm{F}_{10}}$ & $\begin{array}{l}\text { genetic } \\
\text { gain }\end{array}$ \\
\hline GEBV-O & 83.77 & 42.52 & -41.25 & 3.93 & 4.12 & 0.19 \\
\hline GD-O-30 & 89.50 & 49.69 & -39.81 & 3.86 & 4.19 & 0.33 \\
\hline GD-O-50 & 90.11 & 50.13 & -39.98 & 3.80 & 4.14 & 0.34 \\
\hline GD-O-100 & 92.10 & 52.10 & -40.00 & 3.64 & 4.08 & 0.44 \\
\hline GEBV-GD-30 & 87.26 & 42.99 & -44.27 & 3.90 & 4.22 & 0.32 \\
\hline GEBV-GD-50 & 87.95 & 43.50 & -44.45 & 3.84 & 4.21 & 0.37 \\
\hline GEBV-GD-100 & 89.27 & 43.95 & -45.32 & 3.70 & 4.17 & 0.47 \\
\hline
\end{tabular}


554 (i) $\overline{G E B V}_{P}$ : the GEBV average among the 10 selected parental lines. $\overline{G E B V}_{F_{10}}$ : the 555 GEBV average among the resulting $2700 \mathrm{~F}_{10}$ RILs.

556 (ii) GEBV-O: the subset of the top 10 accessions with the minimal or maximal GEBVs; 557 GD-O-30, -50, -100: the subsets of 10 accessions with the maximal D-scores chosen 558 from the candidate sets composed of the top 30, 50, and 100 accessions, respectively; 559 GEBV-GD-30, -50, -100: the subsets of the top 2 accessions plus 8 accessions chosen

560 from the remainder of the candidate sets composed of the top 30, 50, and 100 accessions, 561 respectively, which have the maximal D-scores.

562 (iii) BRSW: brown rice seed width; FPP: florets per panicle; FTAA: flowering time at 563 Arkansas; FTAF: flowering time at Faridpur; PH: plant height; PNPP: panicle number 564 per plant. 
597 Table 5: The average of genetic gains from the 30 repetitions for Dataset II.

598

599

\begin{tabular}{|c|c|c|c|}
\hline & \multicolumn{3}{|c|}{ YLD } \\
\hline & $\overline{\mathrm{GEBV}}_{\mathrm{P}}$ & $\overline{\mathrm{GEBV}}_{\mathrm{F}_{10}}$ & $\begin{array}{l}\text { genetic } \\
\text { gain }\end{array}$ \\
\hline GEBV-O & 5571.61 & 6468.60 & 896.99 \\
\hline GD-O-30 & 5452.39 & 6488.02 & 1035.63 \\
\hline GD-O-50 & 5436.58 & 6484.58 & 1048.00 \\
\hline GD-O-100 & 5289.74 & 6540.72 & 1250.98 \\
\hline GEBV-GD-30 & 5538.44 & 6501.23 & 962.79 \\
\hline GEBV-GD-50 & 5522.45 & 6482.13 & 959.68 \\
\hline \multirow[t]{3}{*}{ GEBV-GD-100 } & 5454.37 & 6535.79 & 1081.42 \\
\hline & \multicolumn{3}{|c|}{$\mathrm{PH}$} \\
\hline & $\overline{\mathrm{GEBV}}_{\mathrm{P}}$ & $\overline{\mathrm{GEBV}}_{\mathrm{F}_{10}}$ & $\begin{array}{l}\text { genetic } \\
\text { gain }\end{array}$ \\
\hline GEBV-O & 97.75 & 85.89 & -11.86 \\
\hline GD-O-30 & 102.20 & 87.59 & -14.61 \\
\hline GD-O-50 & 103.66 & 89.99 & -13.67 \\
\hline GD-O-100 & 106.83 & 91.85 & -14.98 \\
\hline GEBV-GD-30 & 99.00 & 86.01 & -12.99 \\
\hline GEBV-GD-50 & 99.39 & 85.99 & -13.40 \\
\hline \multirow[t]{3}{*}{ GEBV-GD-100 } & 101.15 & 86.13 & -15.02 \\
\hline & \multicolumn{3}{|c|}{ FT } \\
\hline & $\overline{\mathrm{GEBV}}_{\mathrm{P}}$ & $\overline{\mathrm{GEBV}}_{\mathrm{F}_{10}}$ & $\begin{array}{l}\text { genetic } \\
\text { gain }\end{array}$ \\
\hline GEBV-O & 83.14 & 77.84 & -5.30 \\
\hline GD-O-30 & 83.98 & 78.73 & -5.25 \\
\hline GD-O-50 & 84.57 & 78.19 & -6.38 \\
\hline GD-O-100 & 85.62 & 78.39 & -7.23 \\
\hline GEBV-GD-30 & 83.44 & 77.90 & -5.54 \\
\hline GEBV-GD-50 & 83.69 & 77.76 & -5.93 \\
\hline GEBV-GD-100 & 84.16 & 77.89 & -6.27 \\
\hline
\end{tabular}

601 (i) $\overline{G E B V}_{P}$ : the GEBV average among the 10 selected parental lines. $\overline{G E B V}_{F_{10}}$ : the 
602 GEBV average among the resulting $2700 \mathrm{~F}_{10}$ RILs.

603 (ii) GEBV-O: the subset of the top 10 accessions with the minimal or maximal GEBVs;

604 GD-O-30, -50, -100: the subsets of 10 accessions with the maximal D-scores chosen 605 from the candidate sets composed of the top 30, 50, and 100 accessions, respectively; 606 GEBV-GD-30, -50, -100: the subsets of the top 2 accessions plus 8 accessions chosen 607 from the remainder of the candidate sets composed of the top 30, 50, and 100 accessions, 608 respectively, which have the maximal D-scores.

609 (iii) YLD: yield; PH: plant height; FT: flowering time.

610

611

612

613

614

615

616

617

618

619

620 


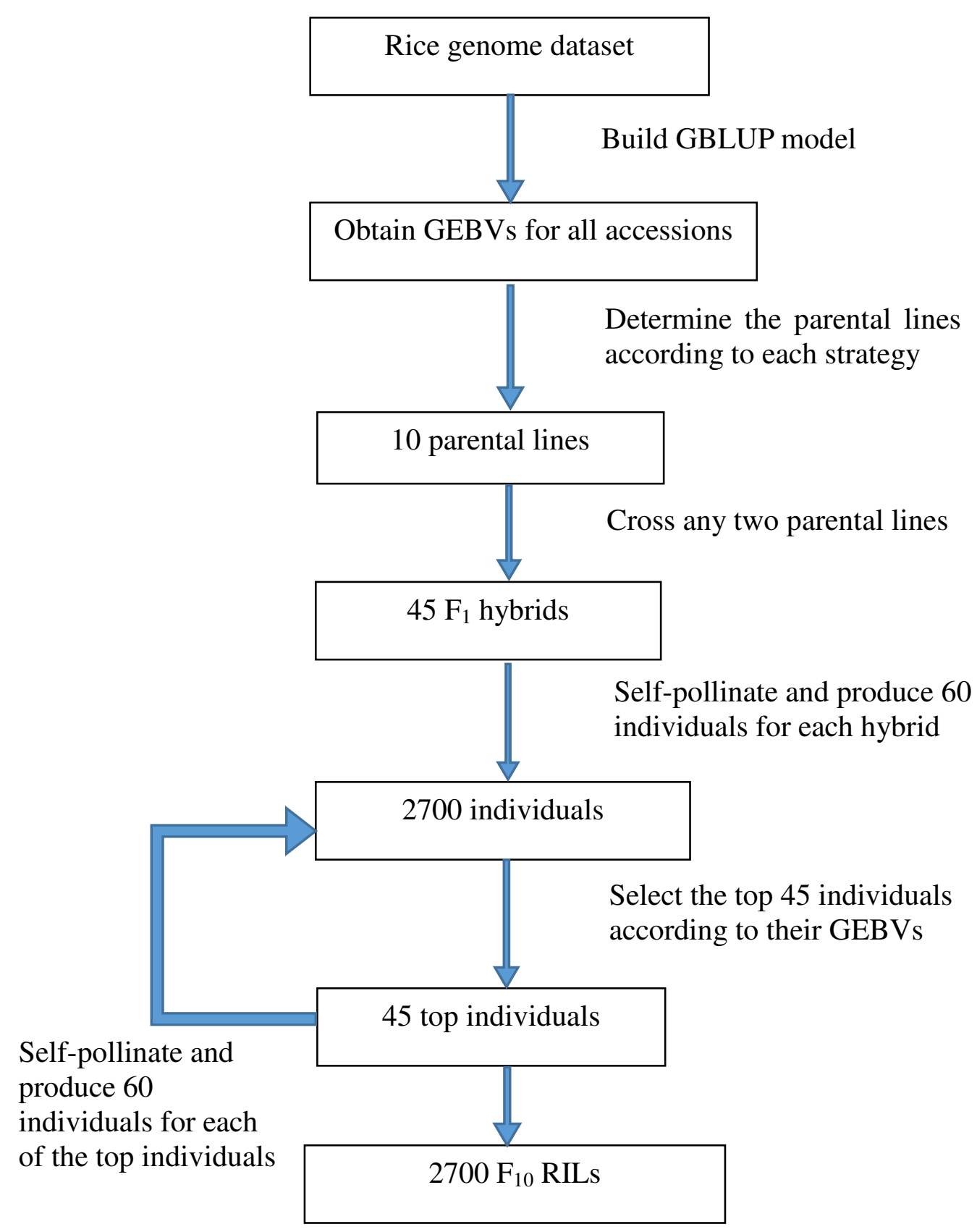

622 Figure 1: The working flow for the Monte Carlo simulation.

623 GEBV: genomic estimated breeding value; GBLUP: genomic best linear unbiased

624 predictor; RIL: recombinant inbred line. 
BRSW (the larger the better)

$\rightarrow$ (i).GEBV-0 $\rightarrow$ (ii).GD-O-30 $\rightarrow$ (iii).GD-O-50 $\rightarrow$ (iv).GD-O-100

$\rightarrow$ (v).GEBV-GD-30 $\rightarrow$ (vi).GEBV-GD-50 $\rightarrow$ (vii).GEBV-GD-100

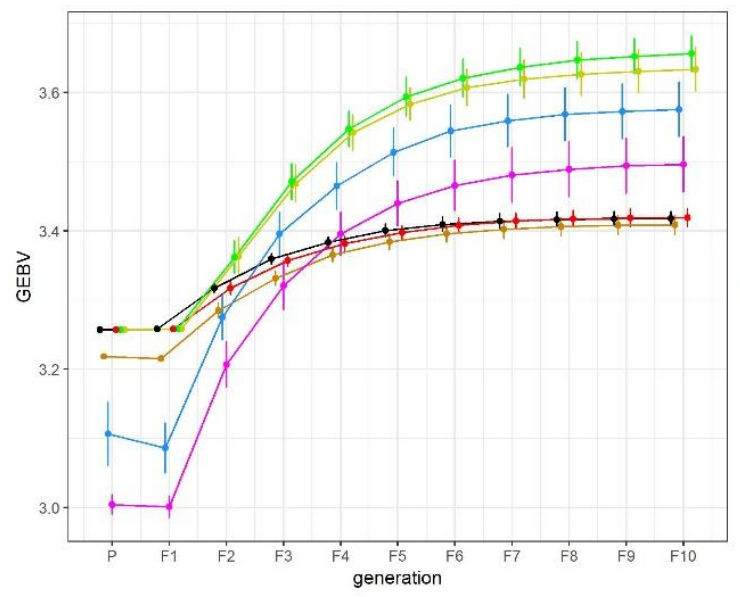

626

FTAA (the smaller the better)

$\rightarrow$ (i).GEBV-0 $\rightarrow$ (ii).GD-O-30 $\rightarrow$ (ii).GD-0-50 $\rightarrow$ (iv).GD-O-100

$\rightarrow$ (v).GEBV-GD-30 $\rightarrow$ (vi).GEBV-GD-50 $\rightarrow$ (vi).GEBV-GD-100

627

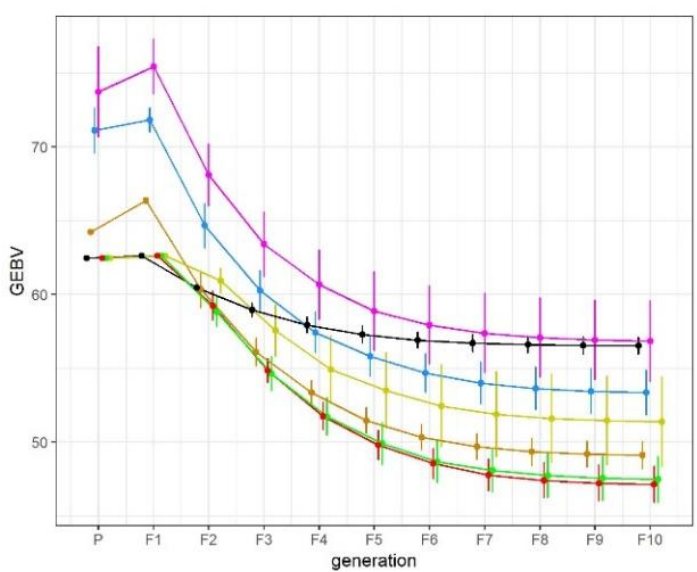

$\mathrm{PH}$ (the smaller the better)

$\rightarrow$ (i).GEBV-0 $\rightarrow$ (ii).GD-O-30 $\rightarrow$ (iii).GD-O-50 $\rightarrow$ (iv).GD-O-100

$\rightarrow$ (v).GEBV-GD-30 $\rightarrow$ (vi).GEBV.GD-50 $\rightarrow$ (vii).GEBV-GD-100

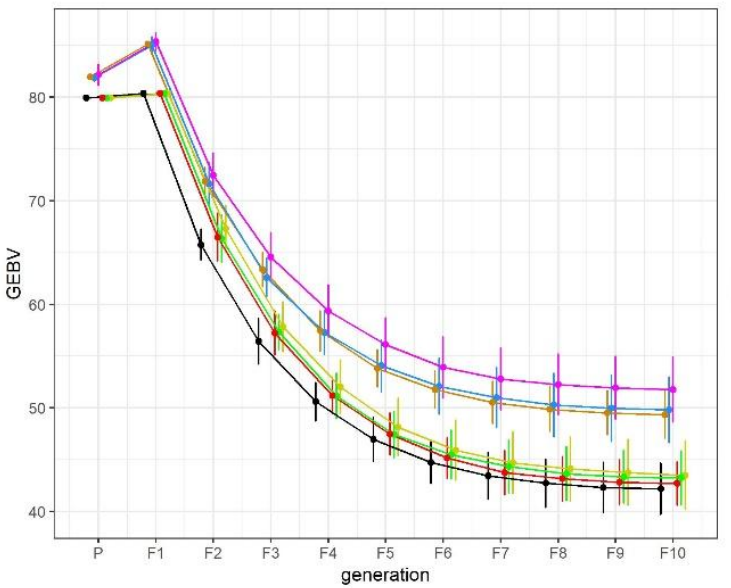

FPP (the larger the better)

$\rightarrow$ (i).GEBV-0 $\rightarrow$ (ii).GD-O-30 $\rightarrow$ (iii).GD-O-50 $\rightarrow$ (iv).GD-0-100

$\rightarrow$ (v).GEBV-GD-30 $\rightarrow$ (vi).GEBV-GD-50 $\rightarrow$ (vii).GEBV-GD-100

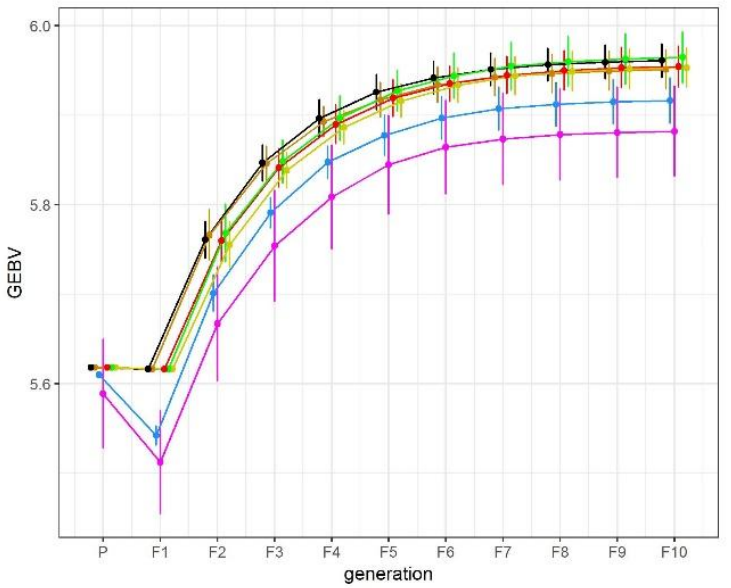

FTAF (the smaller the better)

$\rightarrow$ (i).GEBV-0 $\rightarrow$ (ii).GD-O-30 $\rightarrow$ (iii).GD-O-50 $\rightarrow$ (iv),GD-O-100

$\rightarrow$ (v).GEBV-GD-30 $\rightarrow$ (vi),GEBV-GD-50 $\rightarrow$ (vii).GEBV-GD-100

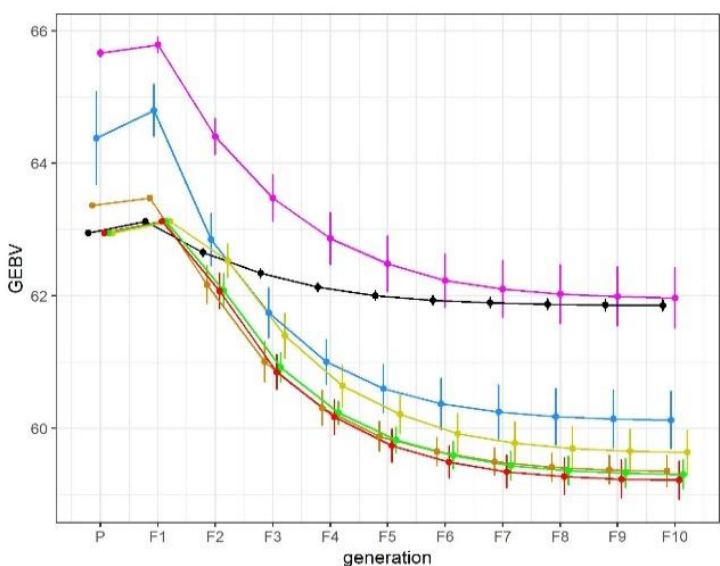

PNPP (the larger the better)

$\rightarrow$ (i).GEBV $-0 \quad \rightarrow$ (ii).GD-O-30 $\rightarrow$ (iii),GD-O-50 $\rightarrow$ (iv).GD-O-100

$\rightarrow(v)$.GEBV-GD-30 $\rightarrow$ (vi).GEBV-GD-50 $\rightarrow$ (vi).GEBV-GD-100

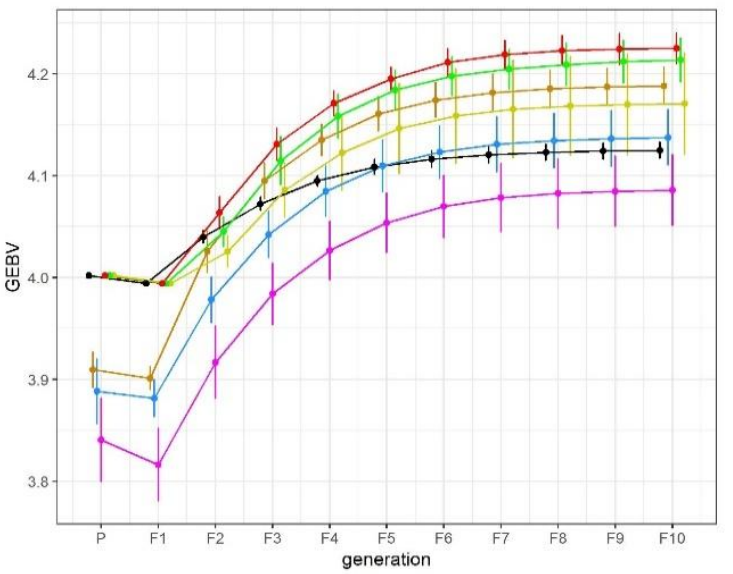


629 Figure 2: The GEBV averages for the best individuals from the 30 repetitions at 630 consecutive generations for the six chosen traits in Dataset I.

631 (i) GEBV-O: the subset of the top 10 accessions with the minimal or maximal GEBVs; 632 GD-O-30, -50, -100: the subsets of 10 accessions with the maximal D-scores chosen 633 from the candidate sets composed of the top 30, 50, and 100 accessions, respectively; 634 GEBV-GD-30, -50, -100: the subsets of the top 2 accessions plus 8 accessions chosen 635 from the remainder of the candidate sets composed of the top 30, 50, and 100 accessions, 636 respectively, which have the maximal D-scores.

637 (ii) BRSW: brown rice seed width; FPP: florets per panicle; FTAA: flowering time at 638 Arkansas; FTAF: flowering time at Faridpur; PH: plant height; PNPP: panicle number 639 per plant.

640

641

642

643 
YLD. (the larger the better)

$\rightarrow$ (i).GEBV-O $\rightarrow$ (ii).GD-O-30 $\rightarrow$ (iii).GD-0-50 (iv)-GD-0-100

$\rightarrow$ (v).GEBV-GD-30 - (vi).GEBV-GD-50 - (vii).GEBV-GD-100

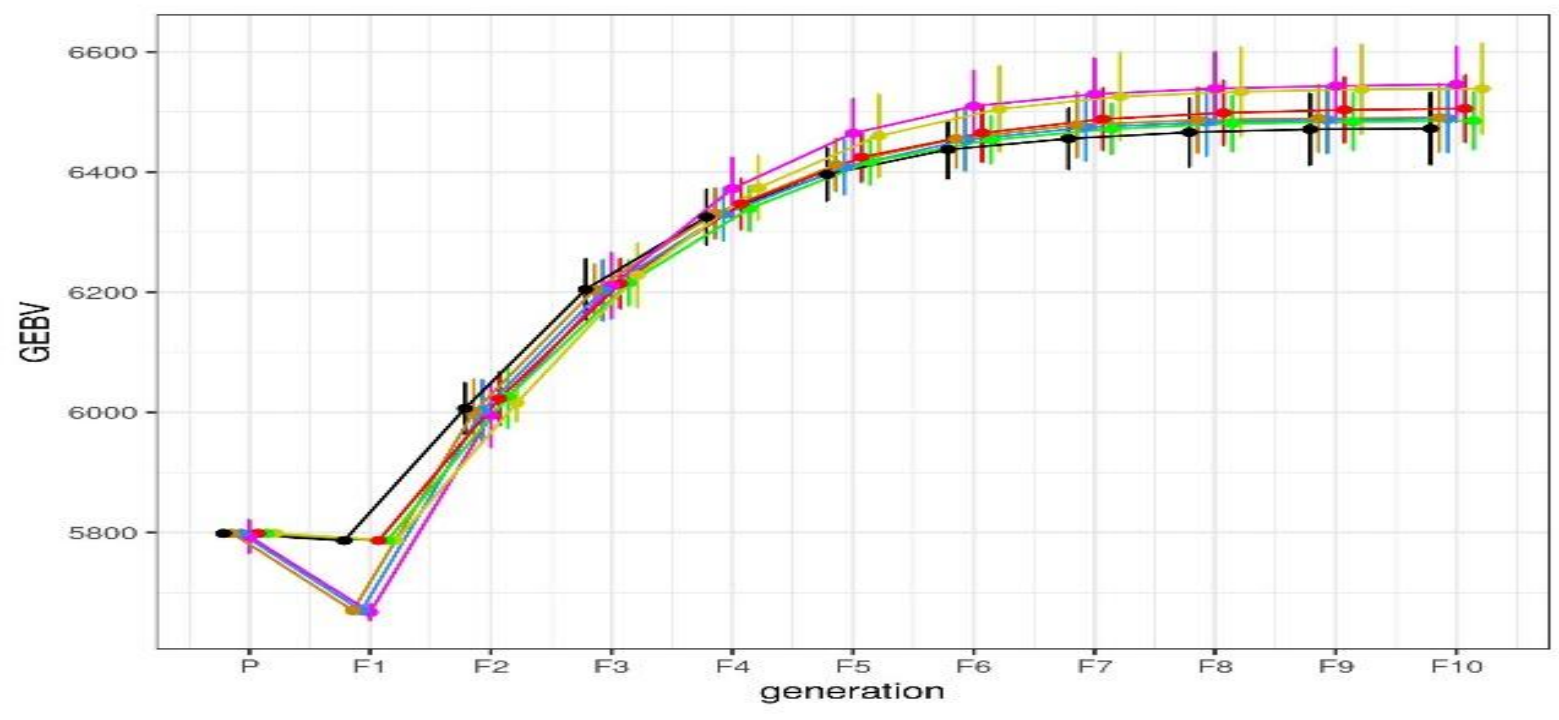

645

$\mathrm{PH}$. (the smaller the better)
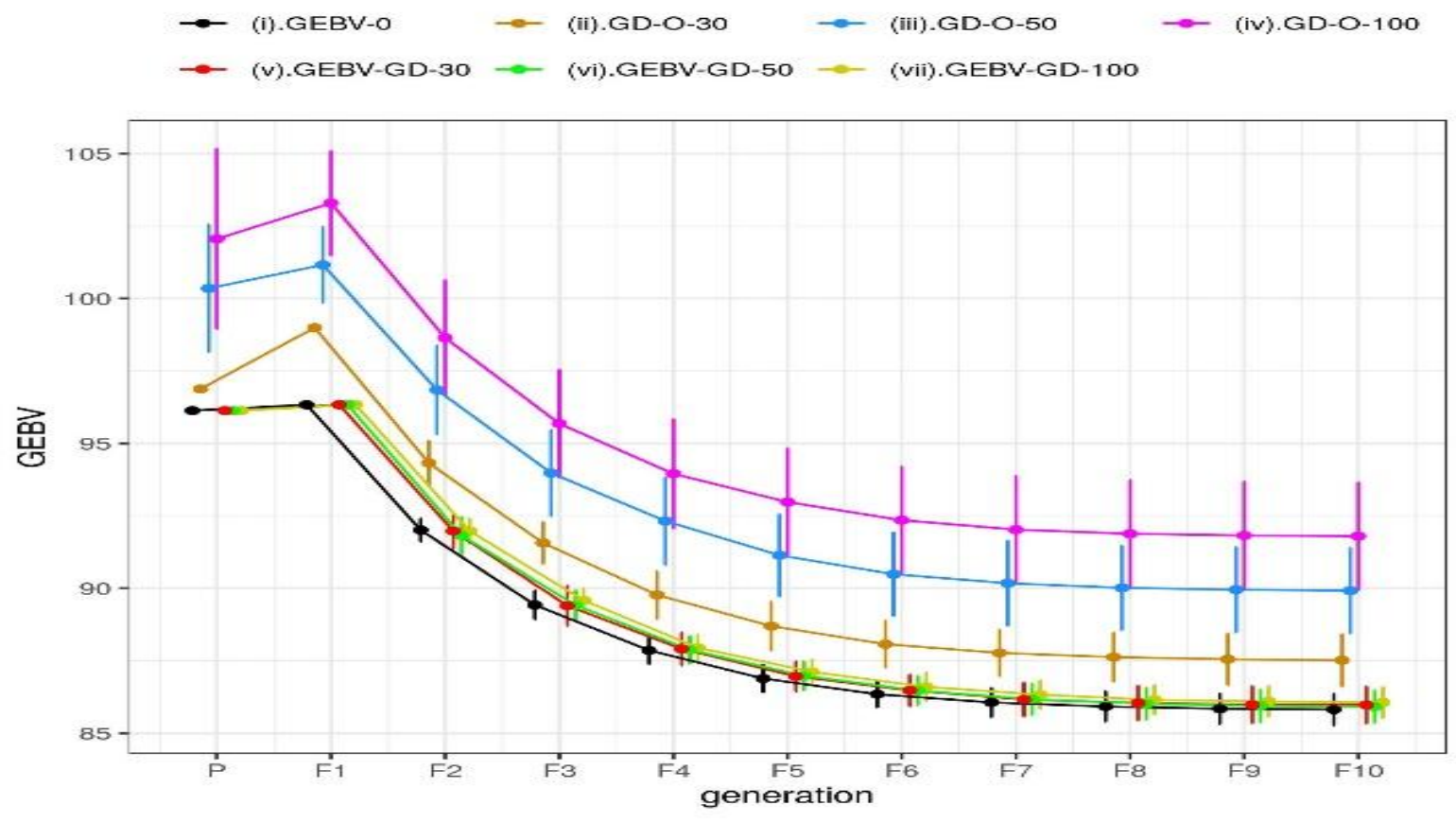
FT. (the smaller the better)

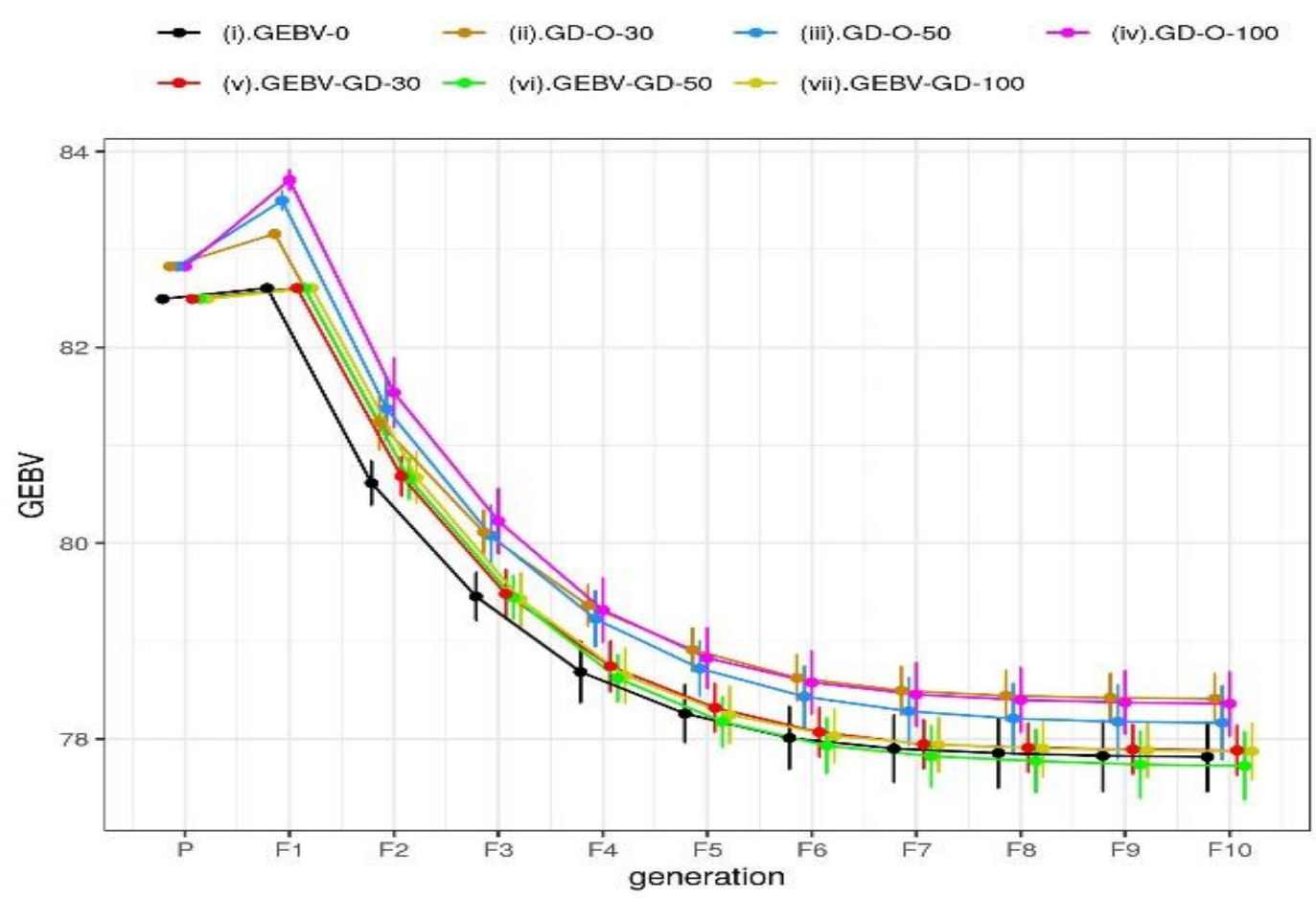

647

648 Figure 3: The GEBV averages for the best individuals form the 30 repetitions at 649 consecutive generations for the three target traits in Dataset II.

650 (i) GEBV-O: the subset of the top 10 accessions with the minimal or maximal GEBVs; 651 GD-O-30, -50, -100: the subsets of 10 accessions with the maximal D-scores chosen 652 from the candidate sets composed of the top 30, 50, and 100 accessions, respectively; 653 GEBV-GD-30, -50,-100: the subsets of the top 2 accessions plus 8 accessions chosen 654 from the remainder of the candidate sets composed of the top 30, 50, and 100 accessions, 655 respectively, which have the maximal D-scores.

656 (ii) YLD, yield; PH, plant height; FT, flowering time. 
Figures

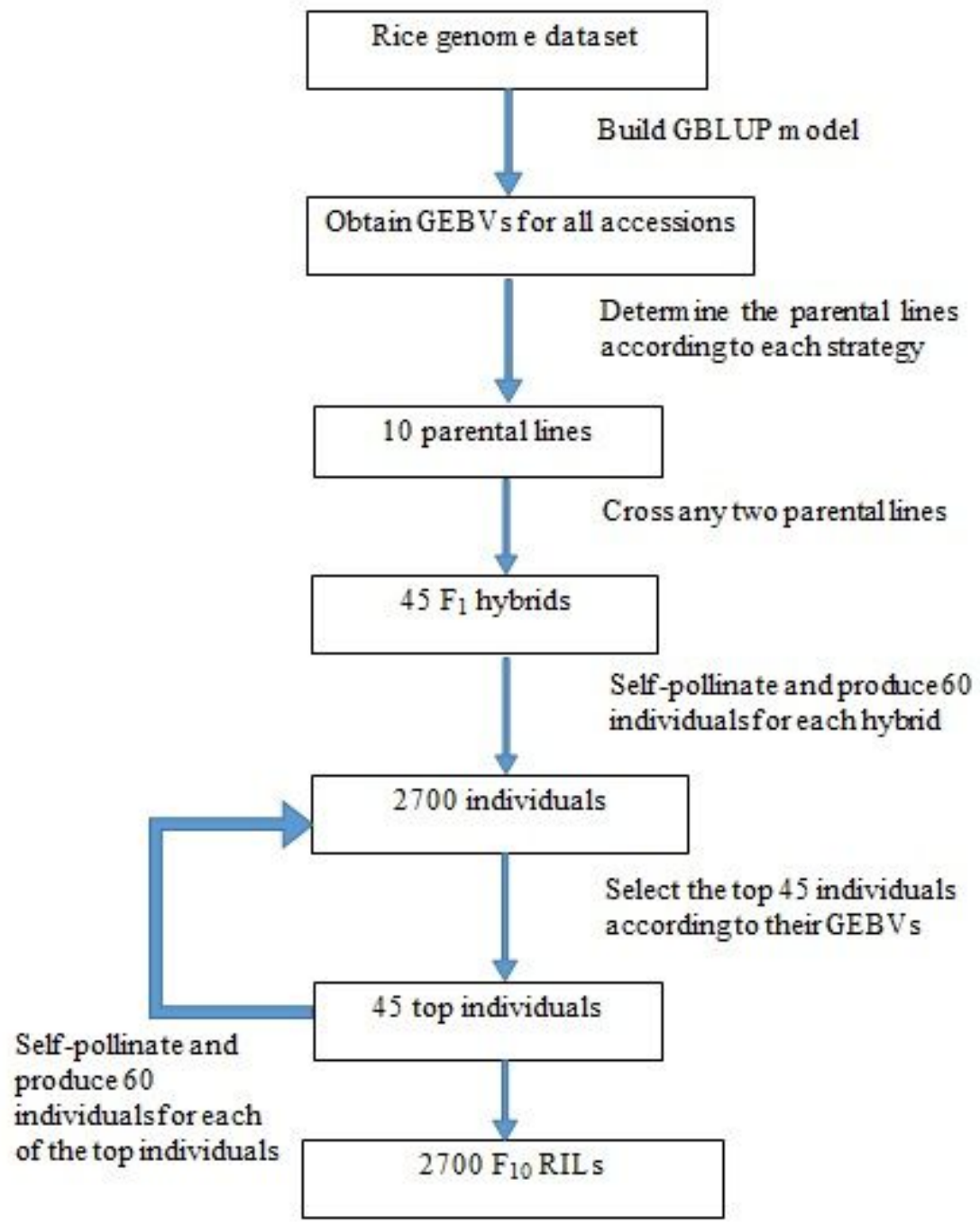

\section{Figure 1}

The working flow for the Monte Carlo simulation. GEBV: genomic estimated breeding value; GBLUP: genomic best linear unbiased predictor; RIL: recombinant inbred line. 

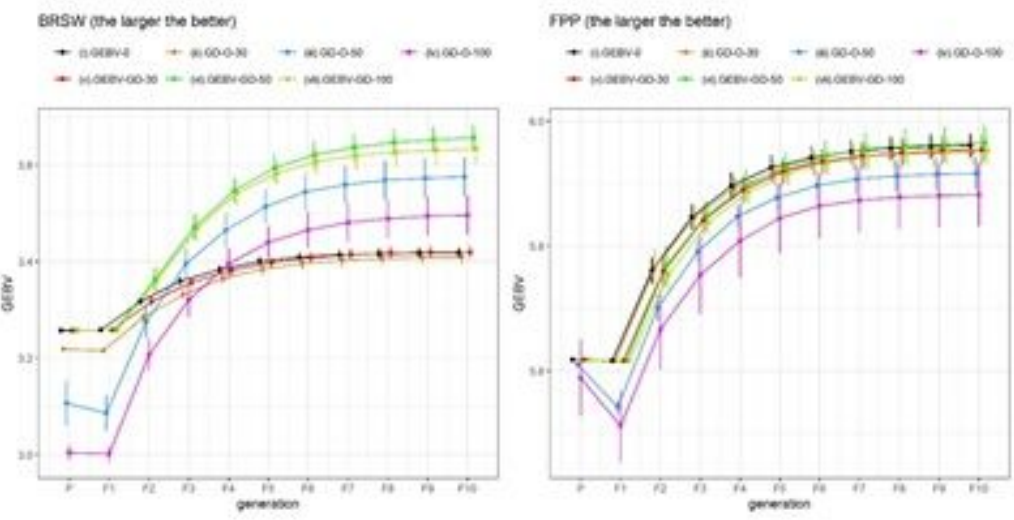

FIAA ane smater the beter?

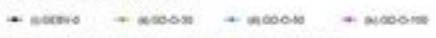

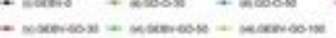

FTAF the smater ne teosen

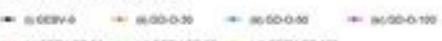

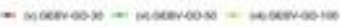
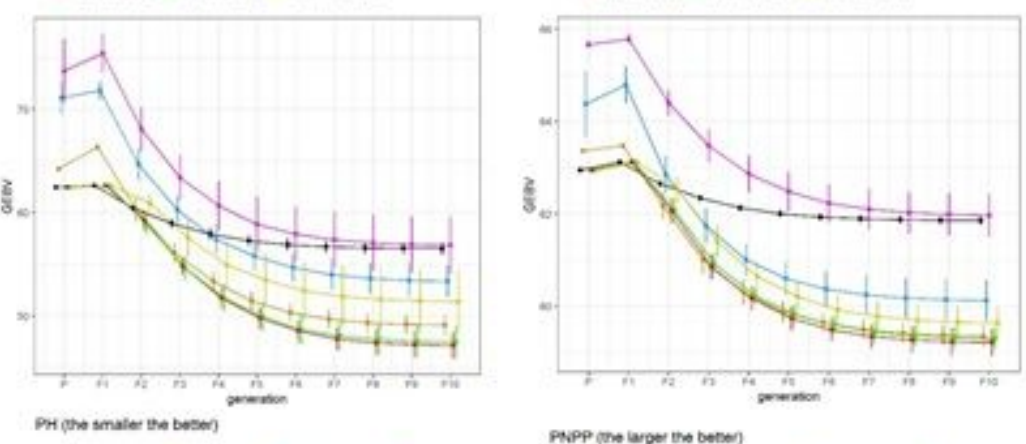

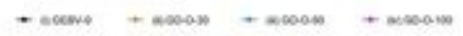

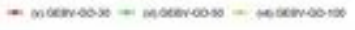

PAipp (the lapper the betsen)

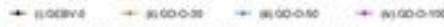

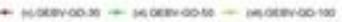
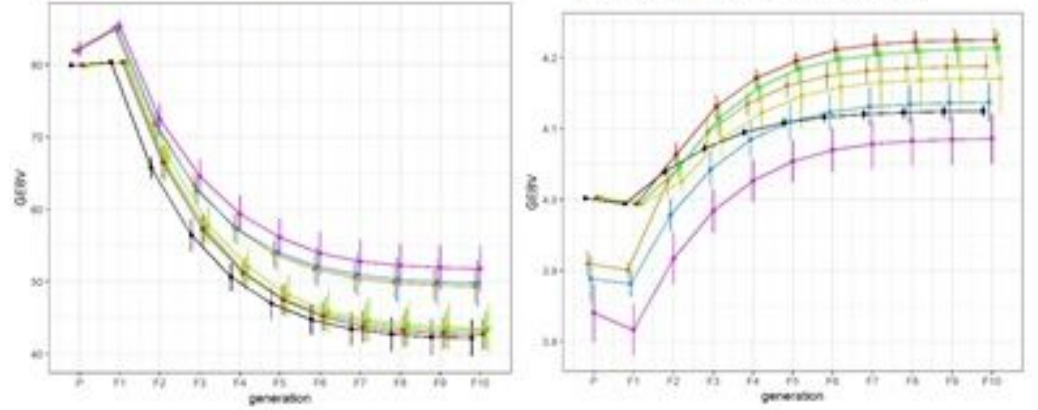

Figure 2

The GEBV averages for the best individuals from the 30 repetitions at consecutive generations for the six chosen traits in Dataset I. (i) GEBV-O: the subset of the top 10 accessions with the minimal or maximal GEBVs; GD-0-30, -50, -100: the subsets of 10 accessions with the maximal D-scores chosen from the candidate sets composed of the top 30,50, and 100 accessions, respectively; GEBV-GD-30, $-50,-100$ : the subsets of the top 2 accessions plus 8 accessions chosen from the remainder of the candidate sets composed of the top 30, 50, and 100 accessions, respectively, which have the maximal D-scores. (ii) BRSW: brown rice seed width; FPP: florets per panicle; FTAA: flowering time at Arkansas; FTAF: flowering time at Faridpur; PH: plant height; PNPP: panicle number per plant. 

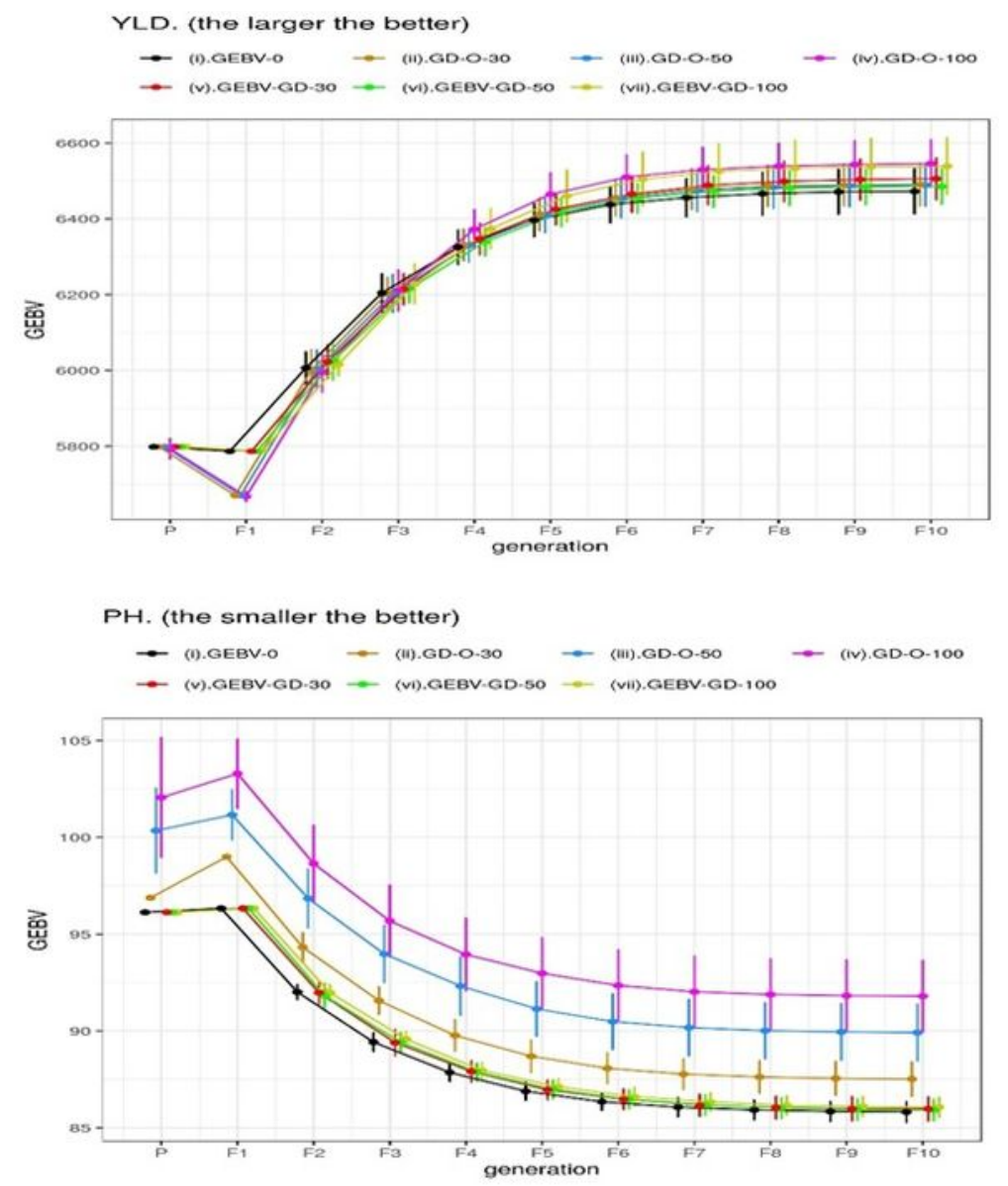

FT. (the smaller the better)

$$
\rightarrow \text { (i).GEBV-0 } \rightarrow \text { (ii).CD.0-30 } \rightarrow \text { (ii).GD.0.50 } \rightarrow \text { (iv).GD.0.100 }
$$

$\rightarrow$ (v).GEBV-GD-30 - (v).GEBV-GD-50 - (vii).GEBV-GD-100

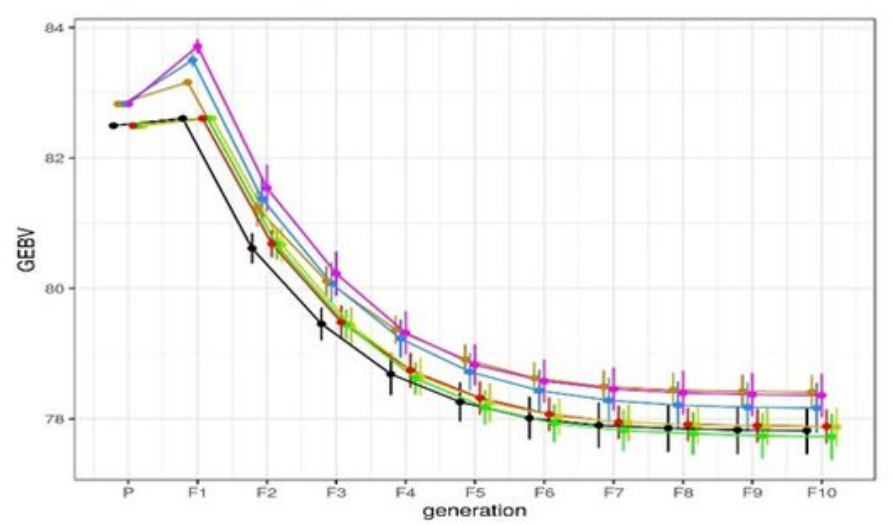

\section{Figure 3}

The GEBV averages for the best individuals form the 30 repetitions at consecutive generations for the three target traits in Dataset II. (i) GEBV-O: the subset of the top 10 accessions with the minimal or maximal GEBVs; GD-0-30, -50, -100: the subsets of 10 accessions with the maximal D-scores chosen from the candidate sets composed of the top 30, 50, and 100 accessions, respectively; GEBV-GD-30, -50, -100: the subsets of the top 2 accessions plus 8 accessions chosen from the remainder of the candidate 
sets composed of the top 30,50, and 100 accessions, respectively, which have the maximal D-scores. (ii) YLD, yield; $\mathrm{PH}$, plant height; $\mathrm{FT}$, flowering time. 